\title{
Evaluating the Impact of a New Smartphone Texting Tool on Patient Care in Obstetrics, an Emergent Healthcare Setting
}

\author{
Jacqueline Feinberg ${ }^{1}$ Sara Shaw ${ }^{2}$ Nitu Kashyap ${ }^{1}$ Jessica Illuzzi ${ }^{1} \quad$ Katherine Campbell $^{1}$ \\ Allen L. Hsiao ${ }^{1}$ Christian M. Pettker ${ }^{1}$ \\ ${ }^{1}$ Department of Obstetrics and Gynecology, Yale School of Medicine, \\ New Haven, Connecticut, United States \\ 2 Yale New Haven Hospital, New Haven, Connecticut, United States \\ Address for correspondence Jacqueline Feinberg, MD, Department of \\ Obstetrics and Gynecology, Yale School of Medicine, 333 Cedar Street, \\ New Haven, Connecticut 06510, United States \\ (e-mail: feinberj@mskcc.org).
}

Appl Clin Inform 2019;10:879-887.

\begin{abstract}
Keywords

- text messaging

- smart phone

- hospital system

- obstetrics

Background Hospitals across the country are investing millions of dollars to adopt new Health Insurance Portability and Accountability Act (HIPAA)-compliant secure text messaging systems. However, in nearly all cases, these implementations are occurring without evaluation of their impact on patient care.

Objective To evaluate perceived impact on patient care and workflow of new text messaging system implemented in obstetrics at Yale-New Haven Hospital and to inform guidelines for future implementations in emergent settings.

Methods A new HIPAA-compliant texting system was implemented in obstetrics in 2016. Before implementation of the new system, residents and nurses were surveyed on perceived effect of communication system (pagers with text receiving, service mobile phones, personal cell phones) on clinical workflow and patient care using 5point Likert scale and open-ended questions. Following roll-out ( 1 and 6 months), both teams were surveyed with same questions. Results were compared using WilcoxonMann-Whitney test ( $0-1$ months and then $0-6$ months). Open-ended question results were qualitatively compared for recurrent unifying themes.

Results In both nursing and resident domains, 1 month after implementation, the new communication system was perceived to significantly improve efficiency and patient care across all metrics. After 6 months, this effect decayed in nearly all categories (including efficiency, real-time communication, and knowledge of covering provider). The exception was nurse's knowledge of which resident to contact and resident's timely evaluation of patient, for which we observed sustained improvements. System shortcomings identified included interrupted connection (i.e., dropped calls), dysfunctional and inaccurate alert system, and unclear identification of the covering provider.

Conclusion A new text-messaging-based communication system may improve efficiency and patient care in emergent settings, but system shortcomings can substantially erode potential benefits over time. We recommend implementers evaluate new systems for a set of specific functional requirements to increase probability of sustained improvement and decrease risk of poor patient outcomes.
\end{abstract}

received

May 30, 2019

accepted after revision

September 18, 2019 (c) 2019 Georg Thieme Verlag KG Stuttgart · New York
DOI https://doi.org/

$10.1055 / \mathrm{s}-0039-1700868$

ISSN 1869-0327. 


\section{Background and Significance}

In response to the ubiquity of text (short message service [SMS]) messaging among clinicians ${ }^{1}$ and calls for userfriendly messaging systems in healthcare, ${ }^{2}$ hospitals around the country are investing millions of dollars to adopt new Health Insurance Portability and Accountability Act (HIPAA)compliant secure messaging systems. Their goal is to improve security, privacy, and patient care. These new systems have the potential to change communication patterns among clinical team, responsiveness to emergent situations, and more. However, in nearly all cases, these implementations are occurring without evaluation of their impact on patient care. This is particularly important in emergent clinical fields, such as obstetrics, where clinical status can rapidly change, where delay in care can result in negative outcomes, and where multiple systems to communicate patient status are relied on including pagers and mobile phones. In the case of obstetrics, prolonged time to delivery impacts outcomes in fetal bradycardia and cord prolapse, as well as shoulder dystocia, postpartum hemorrhage, and eclamptic seizures. ${ }^{3-8}$ As such, changing clinical workflow without evaluation is not dissimilar to adopting a new medical device without trials.

Few studies have been conducted to evaluate the impact of new secure messaging systems on perceived or potential outcomes; we found only one that has been conducted within an emergent field. ${ }^{9-16}$ Furthermore, the American College of Obstetricians and Gynecologists' (ACOG) recent committee opinion calls for guidelines, checklists, and protocols across clinical care. ${ }^{17}$ Broadly within the medical landscape, generalized frameworks exist for optimal implementation of healthcare information technology (IT). However, specifically within obstetrics or other emergent fields and in the realm of text messaging-based communication, none have been reported. ${ }^{18}$ As such, it is equally important to evaluate implementations for lessons learned, in an effort to develop guidelines specific to the unique requirements of emergent care in obstetrics.

\section{Objectives}

In 2016, Yale-New Haven Hospital implemented a new HIPAA-compliant secure communication platform in obstetrics. In this study, we evaluate resident and nurse perceptions regarding patient care and workflow after implementation. Our hypothesis was that all domains evaluated will show interval improvements after system implementation. In this study, we also leverage our findings in the context of general guidelines for implementation of healthcare IT to propose emergent care and obstetrics-specific recommendations that can be utilized by other hospital systems.

\section{Methods}

This study was performed on the obstetrics unit at a tertiary care academic hospital located in Connecticut, with approx- imately 4,600 deliveries each year. Each unit is staffed by residents specializing in obstetrics and gynecology, fellows specializing in maternal-fetal medicine, attendings in both specialties, as well as registered nurses assigned to shifts on the labor and delivery, antepartum, or postpartum floors.

Prior to this intervention, non-face-to-face communication occurred via pagers with text receiving, service mobile phones, and personal cell phones. In the majority of clinical scenarios, one-way $\alpha$-numeric text pages could be sent and role-based phone lines were used. In emergency scenarios, alerts were sent synchronously to both the role-based phone lines of nurses and residents and to their pagers.

MH-Cure (a product of Mobile Heartbeat), a HIPAAcompliant secure messaging system designed for use in a hospital system, was launched in the obstetrics unit in August 2016 (there are numerous similar mobile applications currently on the market, and the authors have no conflicts of interest with this product). Phone calls and secure messages could now be sent and received between any actively logged-in users. After logging-in, users assign themselves to a hospital floor, a role when relevant (e.g., "charge nurse for labor and delivery," "covering resident for high-risk obstetrics"), and can assign themselves to specific patients. The system receives information from Epic, the electronic health record in use at the hospital to populate unit-specific data and laboratory results but does not transmit information back (i.e., assignments made in $\mathrm{MH}$-Cure do not appear in Epic). Nurses use shared devices which connect to the hospital wireless fidelity (Wi-Fi) network and are returned at the end of shifts. Residents are each provided a hospital-purchased phone that connects to both cellular LTE network and the hospital's Wi-Fi. The texting interface is similar to commercially available smartphone systems. All written communication between users is archived and discoverable, and it is automatically deleted from each device after 24 hours. There is a built-in emergency alert system that can be activated to alert all users logged onto a specific unit at once.

To maximize the precision and rigor of measurement of the new system's impact, a mixed-methods approach was taken with a survey containing quantitative perception questions followed by qualitative assessment questions. Prior to implementation of the new system (MayJune 2016), residents and nurses were surveyed using convenience sampling (on day, night, and evening shifts and antepartum, labor and delivery, and postpartum units) on perceived effect of current communication system on clinical workflow and patient care. Survey items were based on a review of literature regarding communication practices, pilot-tested with three nurses and three residents for time and understandability, and then modified for broader rollout. ${ }^{9,10}$ Following roll-out, both teams were again surveyed using convenience sampling (after 1 month in September 2016 and after 6 months in February and March 2017) with same questions. Surveys were labeled with a unique participant ID to preserve anonymity. During the study period, survey respondents were subject to change due to resident turnover (study period was over change in resident 
academic year at the end of June/beginning of July) and possible nurse turnover.

Surveys included questions about baseline role-related characteristics and communication habits. Regarding perceived effectiveness of communication systems, questions were split into two categories: workflow and patient care. Questions on workflow addressed the following topics: communication clarity, efficiency, real-time responsiveness, and knowledge of covering provider. Questions on patient care addressed the following: timely evaluation by physicians, resident response time to patient concerns, accuracy of orders (asked only of nurses), and nurses execution of treatment plan (asked only of residents). Above questions were answered using a 5-point Likert scale. To validate and aid in the interpretation of the responses to the survey questions, respondents were also asked two open-ended questions requesting qualitative perception on what the user found to be effective and ineffective in the communication system.

Surveys were analyzed by participant ID and found to have minimal repeated measures, more closely resembling independent sampling. Outcomes were reported as medians and interquartile ranges and compared using the Wilcoxon-
Mann-Whitney test for nonparametric distributions. With at least 16 participants in each survey subgroup, this study was powered to $80 \%$, and a $p$-value of 0.05 to detect one categorical change on a five-point Likert scale with standard deviation of 1. SAS 9.4 was used for statistical analysis. Furthermore, open-ended question results were qualitatively compared for recurrent unifying themes using methods previously described by Curry et al. ${ }^{19}$ The constant comparison method was used while reviewing survey results to catalog key concepts. The concepts were reviewed by two authors (J.F. and S.S.) with prior comparisons to ensure appropriate categorization.

University Institutional Review Board exemption was granted for this study.

\section{Results}

In each phase of the study, 47 nurses were surveyed and 17 residents on average (range: 16-18) were surveyed. Baseline characteristics of nurses and residents are in - Tables 1 and 2 , respectively.

Nurses were well distributed in different shifts and different sections of obstetrics. Number of times needed to

Table 1 Nurse baseline characteristics and communication habits

\begin{tabular}{|c|c|c|c|}
\hline \multirow[b]{2}{*}{ Baseline characteristic } & \multirow[t]{2}{*}{ Pre } & \multicolumn{2}{|l|}{ Post } \\
\hline & & 1 month & 6 months \\
\hline Total participants & 47 & 47 & 41 \\
\hline \multicolumn{4}{|l|}{ Shift (multiple selections allowed) ${ }^{a}$} \\
\hline Day shift & $16(34.0 \%)$ & $21(44.7 \%)$ & $25(61.0 \%)$ \\
\hline Night shift & $33(70.2 \%)$ & $35(74.5 \%)$ & $17(41.5 \%)$ \\
\hline Evening shift & $6(12.8 \%)$ & $2(4.3 \%)$ & $17(41.5 \%)$ \\
\hline \multicolumn{4}{|c|}{ Obstetrics section of work (multiple selections allowed) ${ }^{a}$} \\
\hline Labor and delivery & $31(66.0 \%)$ & $28(59.6 \%)$ & $26(63.4 \%)$ \\
\hline Antepartum unit & $23(49 \%)$ & $22(46.8 \%)$ & $21(51.2 \%)$ \\
\hline Postpartum unit & $11(23.4 \%)$ & $16(34.0 \%)$ & $11(26.8 \%)$ \\
\hline \multicolumn{4}{|c|}{ How many times each day do you need to get in touch with a resident? ${ }^{a}$} \\
\hline 0 & 0 & 0 & 0 \\
\hline $1-5$ & $19(40.4 \%)$ & $22(47.8 \%)$ & $19(47.5 \%)$ \\
\hline $6-10$ & $19(40.4 \%)$ & $16(34.8 \%)$ & $15(37.5 \%)$ \\
\hline $11-15$ & $5(10.6 \%)$ & $3(6.5 \%)$ & $4(10 \%)$ \\
\hline$>16$ & $4(8.5 \%)$ & $5(10.9 \%)$ & $2(5 \%)$ \\
\hline \multicolumn{4}{|c|}{ What methods do you use to get in touch with residents? (multiple selections allowed) ${ }^{a}$} \\
\hline Paging (call pager number or via computer & $42(89.4 \%)$ & $25(53.2 \%)$ & $11(26.8 \%)$ \\
\hline Calling provider phone (service cell phone) & $36(76.6 \%)$ & $13(27.7 \%)$ & $4(9.8 \%)$ \\
\hline Calling personal cell phones & $6(12.8 \%)$ & $5(10.6 \%)$ & $7(17.1 \%)$ \\
\hline Calling provider office/on call line & $6(12.8 \%)$ & $3(6.4 \%)$ & $5(12.2 \%)$ \\
\hline Mobile heartbeat call & $\mathrm{N} / \mathrm{A}$ & $37(78.7 \%)$ & $37(90.2 \%)$ \\
\hline Mobile heartbeat text & $\mathrm{N} / \mathrm{A}$ & $40(85.1 \%)$ & $40(97.6 \%)$ \\
\hline Other & $4(8.5 \%)$ & 0 & $1(2.4 \%)$ \\
\hline
\end{tabular}

${ }^{a}$ Number of times selected (percent of respondents). 
Table 2 Resident baseline characteristics and communication habits

\begin{tabular}{|c|c|c|c|}
\hline \multirow[t]{2}{*}{ Baseline characteristic } & \multirow[t]{2}{*}{ Pre } & \multicolumn{2}{|l|}{ Post } \\
\hline & & 1 month & 6 months \\
\hline Total participants & 18 & 16 & 17 \\
\hline PGY-1 & 5 & 4 & 3 \\
\hline PGY-2 & 2 & 6 & 3 \\
\hline PGY-3 & 5 & 2 & 6 \\
\hline PGY-4 & 6 & 4 & 5 \\
\hline \multicolumn{4}{|c|}{ How many times during the day do you need to get in touch with a nurse? ${ }^{a}$} \\
\hline 0 & 0 & 0 & $1(5.9 \%)$ \\
\hline $1-5$ & $1(5.9 \%)$ & $3(18.8 \%)$ & $6(35.3 \%)$ \\
\hline $6-10$ & $4(23.5 \%)$ & $3(18.8 \%)$ & $5(29.4 \%)$ \\
\hline $11-15$ & $2(11.8 \%)$ & $2(12.5)$ & $3(17.7 \%)$ \\
\hline$>15$ & $10(58.8 \%)$ & $8(50 \%)$ & $2(11.8 \%)$ \\
\hline \multicolumn{4}{|c|}{ How do you usually go about reaching the nurse? (multiple selections allowed) ${ }^{\mathrm{a}}$} \\
\hline Face-to-face & $8(47.1 \%)$ & $7(43.8 \%)$ & $8(47.1 \%)$ \\
\hline Look up number in EMR and call & $15(88.2 \%)$ & $12(75 \%)$ & $9(52.9 \%)$ \\
\hline Call charge nurse to reach individual nurse & $8(47.1 \%)$ & $3(18.8 \%)$ & $1(5.9 \%)$ \\
\hline Text in mobile heartbeat & $\mathrm{N} / \mathrm{A}$ & $12(75 \%)$ & $9(52.9 \%)$ \\
\hline Call in mobile heartbeat & $\mathrm{N} / \mathrm{A}$ & $7(43.8 \%)$ & $1(5.9 \%)$ \\
\hline Other & $2(11.8 \%)$ & 0 & $2(11.8 \%)$ \\
\hline
\end{tabular}

Abbreviation: EMR, electronic medical record.

${ }^{\mathrm{a}}$ Number of times selected (percent of respondents).

communicate with residents did not change significantly with new system (average of seven times/shift) and adoption by 6 months was more than $90 \%$. Residents were well distributed in different years (study was conducted over start of new resident year). Residents reported fewer attempts needed to contact a nurse throughout the day over the study period (going from 13 times/shift prior to implementation to $7.5 /$ shift 6 months postimplementation $(p=0.002))$. Resident adoption was more than $50 \%$ but with a trend of decreased use after 6 months.

- Table 3 shows all details of nurse survey results. Nurses perceived spending on average 3.5 minutes getting in touch with residents, which trended up after implementation to 4.6 minutes, though this was not a statistically significant finding $(p=0.093)$. Nurses reported the amount of time (6 minutes on average) for a resident to respond to them was the same throughout the study period.

Regarding perceived effectiveness, there was statistically significant improvement (all $p<0.05)$ in categories of workflow and patient care between the preimplementation survey and the 1 month postimplementation survey with the exception of communicating thoughts clearly, which remained unchanged $(4, p=0.163)$. We observed overall improvement in workflow from neutral effect (3) to moderately effective ( 4 , $p \leq 0.016$ for communicating efficiently and real time, and $p<0.001$ for reaching covering resident) and on patient care from neutral impact (3) to weakly positive (4) impact $(p=0.002$ for having appropriate orders and $p<0.001$ for other categories). However, at the 6-month postimplementation phase, all of the evaluated categories trended back to baseline and no longer demonstrated any significant difference compared with preimplementation ( $p$-value ranging from 0.077 to 0.540 ). One exception to the decay of gains was in nurse perception on knowing how to reach the covering resident, which remained improved $(p=0.005$ ).

-Table 4 presents all details of resident survey results. Residents perceived spending an average of 2.8 minutes getting in touch with nurses, which trended down after 1 month ( 2 minutes, $p=0.28$ ) but returned back to baseline at 6 months. Regarding perceived effectiveness, there was significant improvement in workflow areas from neutral effect (3) to moderately positive impact (4) after 1 month ( $p=0.006$ for clear, $p=0.044$ for reaching covering nurse, and $p<0.001$ for other categories). However, this effect diminished by 6 months' time and score was back to neutral effect. In the patient care realm, there was change from minor negative impact to minor positive impact (all $p<0.001$ ) at 1 month. Likewise, this effect also diminished in nearly all areas by 6 months. One exception here to the decay of gains at the 6-month mark was in resident timely evaluation of patient $(p=0.017)$.

-Table 5 presents the themes found in the qualitative analysis of the open-ended results. The most significant effective theme extracted was, by a large majority, the benefits of two-way messaging (e.g., "texting is the most 
Table 3 Nurse comparison of pre- and postimplementation on perceived effectiveness of obstetrics communication

\begin{tabular}{|c|c|c|c|c|c|}
\hline \multirow[t]{2}{*}{ Question } & \multirow[t]{2}{*}{ Pre } & \multicolumn{4}{|l|}{ Post } \\
\hline & & 1 month & p-Value & 6 months & p-Value \\
\hline \multicolumn{6}{|c|}{$\begin{array}{l}\text { About how much time do you spend each time getting in touch with each resident (i.e., finding out who is covering, making the } \\
\text { call, etc.)? }\end{array}$} \\
\hline & 3.5 & 3.5 & 0.942 & 4.6 & 0.093 \\
\hline \multicolumn{6}{|c|}{ How long does it take for resident to get back to you? ${ }^{a}$} \\
\hline & 6.8 & 6.2 & 0.353 & 6.7 & 0.827 \\
\hline \multicolumn{6}{|c|}{ Rate the effectiveness of the current communication system in allowing you to: $(1-5 ; 1 \text { as ineffective to } 5 \text { as very effective })^{b}$} \\
\hline Communicate your thoughts clearly & $4(3-4.5)$ & $4(4-5)$ & 0.163 & $4(3-5)$ & 0.239 \\
\hline Communicate your thoughts efficiently & $4(3-4)$ & $4(4-5)$ & 0.016 & $4(3-4)$ & 0.540 \\
\hline Receive/send messages in real time & $3(2-4)$ & $4(3-5)$ & 0.016 & $3(3-4)$ & 0.199 \\
\hline Know how to reach covering resident & $2(2-4)$ & $3(3-4)$ & $<0.001$ & $3(2-4)$ & 0.005 \\
\hline \multicolumn{6}{|c|}{$\begin{array}{l}\text { Rate the impact of the current communication system on your workflow in: }(1-5 ; 1=\text { strongly negative, } 3=\text { neutral, } 5=\text { strongly } \\
\text { positive })^{b}\end{array}$} \\
\hline Timely evaluation of patient by resident & $3(3-4)$ & $4(3-5)$ & $<0.001$ & $4(3-4)$ & 0.360 \\
\hline Responding to patient concerns & $3(2-4)$ & $4(3-5)$ & $<0.001$ & $3.5(3-4)$ & 0.087 \\
\hline Having appropriate orders & $3(2-4)$ & $4(3-5)$ & 0.002 & $3(2-4)$ & 0.077 \\
\hline
\end{tabular}

${ }^{a}$ Minutes, compared using Student's t-test.

${ }^{\mathrm{b}}$ Median (interquartile range), compared using Wilcoxon-Mann-Whitney test.

Table 4 Resident comparison of baseline and postimplementation perceived effectiveness of obstetrics communication

\begin{tabular}{|c|c|c|c|c|c|}
\hline \multirow[t]{2}{*}{ Question } & \multirow[t]{2}{*}{ Pre } & \multicolumn{4}{|l|}{ Post } \\
\hline & & 1 month & $p$-Value & 6 months & p-Value \\
\hline $\begin{array}{l}\text { How long would you say it takes on average to } \\
\text { get in touch with a nurse? }\end{array}$ & 2.8 & 2 & 0.242 & 2.7 & 0.902 \\
\hline \multicolumn{6}{|c|}{ Rate the effectiveness of the current communication system in allowing you to: $(1=\text { not effective; } 5=\text { very effective })^{b}$} \\
\hline Communicate your thoughts clearly & $4(3-4)$ & $4(3.5-5)$ & 0.006 & $4(3-5)$ & 0.429 \\
\hline Communicate your thoughts efficiently & $3(3-4)$ & $4(3.5-5)$ & $<0.001$ & $3(3-4)$ & 0.177 \\
\hline Receive/send messages in real time & $3(1.75-3)$ & $4(3-4)$ & $<0.001$ & $3(3-4)$ & 0.325 \\
\hline Know how to reach covering nurse & $2(1-2)$ & $3(2.5-3.5)$ & 0.044 & $3(2-3)$ & 0.748 \\
\hline \multicolumn{6}{|c|}{$\begin{array}{l}\text { Rate the impact of the current communication system on your workflow in: }(1=\text { strong negative, } 3=\text { neutral, } 5=\text { strong } \\
\text { positive })^{\mathrm{b}}\end{array}$} \\
\hline Timely evaluation of patient & $2(2-3)$ & $4(3.5-4.5)$ & $<0.001$ & $3(2-4)$ & 0.017 \\
\hline Responding to patient concerns & $3(2-3)$ & $4(4-5)$ & $<0.001$ & $3(2-4)$ & 0.054 \\
\hline Carrying out treatment plan & $2(2-4)$ & $4(4-4.5)$ & $<0.001$ & $4(2.75-4)$ & 0.119 \\
\hline
\end{tabular}

a Minutes, compared using Student's t-test.

bedian (interquartile range), compared using Wilcoxon-Mann Whitney test.

efficient way to communicate"). The next most significant category of themes were benefits of real-time communication (e.g., "I feel like we are more in touch") and benefits of direct contact with the covering provider/nurse (e.g., "goes straight to who you need"). The most frequently mentioned themes in the ineffective category were lack of system connectivity (e.g., "drop service," "shuts down, " calls get dropped," lack of usability (e.g., "notifications...do not clear appropriately, "feels like a toy"), and lack of knowing the correct provider/nurse to contact.

\section{Discussion}

We find that a secure messaging system can initially improve perception of workflow and patient care, but if the system does not meet key functional standards, then the improvements can decay within 6 months across most performance metrics. In addition, our findings regarding the system's functional shortcomings have important implications for future selection of new communication systems by emergent settings such as obstetrics. 
Table 5 Narrative responses on effectiveness and ineffectiveness of new HIPAA-compliant two-way messaging system

\begin{tabular}{|c|c|c|c|c|c|c|}
\hline \multirow[t]{2}{*}{ Theme } & \multicolumn{2}{|l|}{ Effective } & \multirow[t]{2}{*}{ Response example } & \multicolumn{2}{|c|}{ Ineffective } & \multirow[t]{2}{*}{ Response example } \\
\hline & 1 month & 6 months & & 1 month & 6 months & \\
\hline Connectivity & $0 \%$ & $0 \%$ & Not applicable & $40 \%$ & $43 \%$ & $\begin{array}{l}\text { "The phones drop } \\
\text { service frequently," } \\
\text { "At times shuts } \\
\text { down without me } \\
\text { knowing its off," } \\
\text { "Call get dropped } \\
\text { frequently" }\end{array}$ \\
\hline $\begin{array}{l}\text { Real-time } \\
\text { communication }\end{array}$ & $20 \%$ & $9 \%$ & $\begin{array}{l}\text { "I feel like we are } \\
\text { more in touch with } \\
\text { the residents" }\end{array}$ & $9 \%$ & $31 \%$ & $\begin{array}{l}\text { "Most worrisome is } \\
\text { how long it some- } \\
\text { times takes for } \\
\text { messages to be de- } \\
\text { livered-it has } \\
\text { caused many mis- } \\
\text { communications, } \\
\text { arguments, and } \\
\text { delays in care" }\end{array}$ \\
\hline $\begin{array}{l}\text { Two-way } \\
\text { messaging }\end{array}$ & $59 \%$ & $60 \%$ & $\begin{array}{l}\text { "Texting is the most } \\
\text { efficient way to } \\
\text { communicate } \\
\text { about small issues } \\
\text { with patients" }\end{array}$ & $0 \%$ & $0 \%$ & $\begin{array}{l}\text { "Texting is nice } \\
\text { when the messages } \\
\text { actually send in real } \\
\text { time" }\end{array}$ \\
\hline System usability & $12 \%$ & $0 \%$ & $\begin{array}{l}\text { "It is easy to use, } \\
\text { there is a lot of info } \\
\text { at your fingertips" }\end{array}$ & $19 \%$ & $33 \%$ & $\begin{array}{l}\text { "Texts and pages } \\
\text { are consistently } \\
\text { delayed. Notifica- } \\
\text { tions come in } \\
\text { batches and do not } \\
\text { clear appropriately. } \\
\text { The app feels like a } \\
\text { toy for how much } \\
\text { we are supposed to } \\
\text { rely on it" }\end{array}$ \\
\hline $\begin{array}{l}\text { Knowing who to } \\
\text { contact }\end{array}$ & $27 \%$ & $9 \%$ & $\begin{array}{l}\text { "Can easily see who } \\
\text { is assigned to a pa- } \\
\text { tient and text/call } \\
\text { that nurse/resident } \\
\text { effectively" }\end{array}$ & $34 \%$ & $57 \%$ & $\begin{array}{l}\text { "Difficult to know } \\
\text { which nurse/resi- } \\
\text { dent to call when } \\
\text { multiple are signed } \\
\text { into MHB" }\end{array}$ \\
\hline Direct contact & $8 \%$ & $23 \%$ & $\begin{array}{l}\text { "Goes straight to } \\
\text { who you need" }\end{array}$ & 0 & 0 & Not applicable \\
\hline $\begin{array}{l}\text { Effectiveness } \\
\text { during } \\
\text { emergencies }\end{array}$ & 0 & 0 & Not applicable & $4 \%$ & 0 & $\begin{array}{l}\text { "Using in an emer- } \\
\text { gency is not easy" }\end{array}$ \\
\hline
\end{tabular}

Of note, in two areas, there were sustained benefits. Specifically, nurses reported improved knowledge of the correct provider to contact; and residents reported improvement in timely evaluation of patients, which is a strong indication of positive impact. Additionally, the qualitative results of the study show that two-way communication capabilities and the ability to use the directory to find any provider or nurse in the hospital were consistently highlighted as positive attributes and perhaps contribute to the few sustained benefits observed. Unfortunately, other sets of qualitative responses align with the potential for bad outcomes. Users were met with inaccurate alerting (e.g., repeat alerts for already received messages; no alerts for new messages), dropped connection (i.e., getting logged out with no notice), and unclear identification of which person to contact-and therefore voiced concerns regarding delays in care (e.g., "Using in an emergency is not easy," "Most worrisome is how long it sometimes takes for messages to be delivered-it has caused many miscommunications, arguments, and delays in care.").

To further contextualize our results, some published research in other inpatient settings has reviewed the impact of text messaging on provider satisfaction, effect work interruption, real-time communication, and information reliability in other domains. ${ }^{9,11,12}$ For example, a recent roll-out of a system in surgical and general medical units at the Hospital of University of Pennsylvania system showed improved communication, workplace efficiency, and even a relative decrease in length of stay. ${ }^{10,13}$ Another study reviewed text messaging on a medicine floor and showed 
an improvement in communication, but found issues related to interruption of workflow, weakened interprofessional relationships, gap in perceived urgency, and an increase in unprofessional behavior. ${ }^{14}$ In a study in a pediatrics hospital after 1 month of implementation, the authors found a decrease in potential communication failures. ${ }^{15}$ In an emergency room setting, a secure messaging system was used for nonemergent consults which showed decreased length of stay and time until consultation. ${ }^{16}$ In all cases though, these studies are small and in early stages. They call for deeper evaluation of these systems, particularly related to impact on clinical workflow and patient care over longer periods of time. To date, as mentioned earlier, there is a scarcity of studies regarding communication within emergent fields like obstetrics, and in particular a gap regarding textmessaging-based communications. ${ }^{20}$

There are limitations inherent in this study. First, this study measures perceived change rather than objective change. While we advocate future studies with objective measures (e.g., from time-stamped time of response to maternal and fetal outcomes), we caution that the expected noise associated with pairing texting data with objective measures in emergent obstetric scenarios potentially makes these less valuable than our higher fidelity subjective metrics. Second, because our evaluation study took place at a large academic obstetrics unit where residents and nurses are often first-line responders to clinical emergencies, our surveyed population focuses on these stakeholders. Other studies will be required in different provider populations to increase probability that proposed functional requirements solve for sustained improvement over time in diverse care settings.

The information observed in our study can inform future implementations of secure messaging systems both obstetrics and in emergent healthcare settings more broadly. To highlight best practices, we can consider lessons learned in the context of a generally recognized framework for healthcare IT implementations as well as lessons learned from prior evaluation studies of multiple secure messaging systems, adoption strategies, and usage. ${ }^{21-25}$ The framework introduced by Rippen et al considers technology, users, environment, outcomes, and temporality. ${ }^{21}$ On the latter four criteria, this system implementation aligned with suggested standards. For example, there was significant user interest in improving the communication system; trainings were done prior to roll-out; key stakeholders were involved from the ground up; the department and hospital leadership strongly supported the implementation of this new product; there was readily available tech support and IT teams prior to and during the roll-out of this tool; and key trainings and engagement were timed with roll-out. This is especially important because a new roll-out of any technology solution alone cannot address broader behavioral shortcomings. Additionally, as discussed in prior studies, a bottom-up approach to implementation was followed, with targeted emails, in-person trainings, and department champions deployed in support of the effort. 23,26
However, the first component of the framework-technology-is where the insights from our analysis indicated multiple problems. At the highest level, key functional requirements were unfulfilled, including unreliable connection and lack of hospital-wide connectivity (i.e., dropped calls, delays in delivering messages). Other identified problems included inaccurate alert system (e.g., repeated alerts for previously received messages, no alert for new messages) and the system failure to clearly identify the covering nurse for each patient. These misses ultimately undermined the positive features like two-way communication and real-time communication. The focus on ensuring the core functional technology infrastructure is satisfactory aligns with guidance summarized by Liu et al as well. They highlighted the importance of addressing these types of problems at the beginning in an evaluation study in which essential and secondary requirements are developed and stratified. $^{22}$

\section{Conclusion}

From our analysis as well as prior studies on the topic, we are able to recommend technology-focused essential functional requirements for text messaging systems in emergent healthcare settings. That is, in addition to strong case for use, buy-in from the highest levels of leadership, smartly timed trainings, and roll-outs, the following functional requirements need to be optimized for:

- Consistent connectivity (no dropped calls).

- Real-time communication (no delays in delivering messages).

- Functional and accurate alert system.

- Two-way secure messaging.

- Message delivery and read receipt (advantage over traditional SMS for closed loop communication).

- Connection with other clinical software to populate patient lists.

- Clearly identifying covering clinical team for each patient and searching by role.

- Group secure messaging.

- Sending picture-based messages.

Based on our study and in response to the national calls for guidelines, we recommend that the above criteria be considered as functional standards to ensure not only effective rollout but sustained improvement in clinical care as a result of new text messaging systems.

\section{Clinical Relevance Statement}

Hospitals across the country are investing millions of dollars to implement new HIPAA-compliant secure text messaging systems. This has impacts on clinical care and outcomes; however, its effect is rarely studied. Therefore, we evaluated the impact of implementation on patient care and found that a new text-messaging based communication system may improve efficiency and perceived outcomes, but systems need to be designed to avoid particular 
shortcomings that can substantially erode potential benefits over time.

\section{Multiple Choice Questions}

1. When implementing a secure messaging system, what is the most critical factor to change in hospital infrastructure?

a. Create storage units to house smart phones.

b. Optimize smart phone connectivity in every area of hospital.

c. Rewiring of hospital to add connected phones to every room.

d. Equipping staff with back-up devices.

Correct Answer: The correct answer is option b, optimize smart phone connectivity in every area of hospital. While multiple hospital-wide infrastructure changes can be made, few are more important than ensuring reliable connection and hospital-wide connectivity. In this study, we found that dropped calls and delays in delivering messages undermined useful features like two-way communication and real-time communication.

2. What key feature must a new secure messaging system include to ensure ongoing benefit?

a. Caller ID.

b. Ability to look up patient information.

c. Accurate and up-to-date alert system.

d. Video chat option.

Correct Answer: The correct answer is option c, accurate and up-to-date alert system. Our study found that the core benefits of a secure messaging system can be undermined if the system is unreliable. Tools such as caller ID and EHR integration are certainly valuable; however, if the system cannot be trusted with alerts, those features will certainly not be enough to carry its success. Inaccurate alerting (e.g., repeat alerts for already received messages; no alerts for new messages) makes the system untrustworthy and can undermine user confidence.

\section{Protection of Human and Animal Subjects}

The study was performed in compliance with the World Medical Association Declaration of Helsinki on Ethical Principles for Medical Research Involving Human Subjects, and was exempted by University Institutional Review Board.

\section{Conflict of Interest}

None declared.

\section{References}

1 Drolet BC. Text messaging and protected health information: What is permitted? JAMA 2017;317(23):2369-2370

2 Thomas K. Wanted: a WhatsApp alternative for clinicians. BMJ 2018;360:k622
3 Leung TY, Chung PW, Rogers MS, Sahota DS, Lao TT-H, Hung Chung TK. Urgent cesarean delivery for fetal bradycardia. Obstet Gynecol 2009;114(05):1023-1028

4 Leung TY, Lao TT. Timing of caesarean section according to urgency. Best Pract Res Clin Obstet Gynaecol 2013;27(02): 251-267

5 Hillemanns P, Hasbargen U, Strauss A, Schulze A, Genzel-Boroviczeny $\mathrm{O}$, Hepp $\mathrm{H}$. Maternal and neonatal morbidity of emergency caesarean sections with a decision-to-delivery interval under 30 minutes: evidence from 10 years. Arch Gynecol Obstet 2003; 268(03):136-141

6 Schauberger CW. Decision-to-incision times and maternal and infant outcomes. Obstet Gynecol 2006;108(05):1298

7 Prabulos AM, Philipson EH. Umbilical cord prolapse. Is the time from diagnosis to delivery critical? J Reprod Med 1998;43(02): 129-132

8 Bujold E, Francoeur D. Neonatal morbidity and decision-delivery interval in patients with uterine rupture. JObstet Gynaecol Can 2005;27(07):671-673

9 Przybylo JA, Wang A, Loftus P, Evans KH, Chu I, Shieh L. Smarter hospital communication: secure smartphone text messaging improves provider satisfaction and perception of efficacy, workflow. J Hosp Med 2014;9(09):573-578

10 Patel N, Siegler JE, Stromberg N, Ravitz N, Hanson CW. Perfect storm of inpatient communication needs and an innovative solution utilizing smartphones and secured messaging. Appl Clin Inform 2016;7(03):777-789

11 Nguyen C, McElroy LM, Abecassis MM, Holl JL, Ladner DP. The use of technology for urgent clinician to clinician communications: a systematic review of the literature. Int J Med Inform 2015;84(02): 101-110

$12 \mathrm{Wu}$ RC, Tran K, Lo V, et al. Effects of clinical communication interventions in hospitals: a systematic review of information and communication technology adoptions for improved communication between clinicians. Int J Med Inform 2012;81(11): 723-732

13 Patel MS, Patel N, Small DS, et al. Change in length of stay and readmissions among hospitalized medical patients after inpatient medicine service adoption of mobile secure text messaging. J Gen Intern Med 2016;31(08):863-870

$14 \mathrm{Wu} \mathrm{R}$, Rossos P, Quan S, et al. An evaluation of the use of smartphones to communicate between clinicians: a mixed-methods study. J Med Internet Res 2011;13(03):e59

15 Hansen JE, Lazow M, Hagedorn PA. Reducing interdisciplinary communication failures through secure text messaging: a quality improvement project. Pediatr Qual Saf 2018;3(01): e053

16 Gulacti U, Lok U. Comparison of secure messaging application (WhatsApp) and standard telephone usage for consultations on length of stay in the ED. A prospective randomized controlled study. Appl Clin Inform 2017;8(03):742-753

17 Committee on Patient Safety and Quality Improvement. Committee opinion no. 629: clinical guidelines and standardization of practice to improve outcomes. Obstet Gynecol 2015;125(04): 1027-1029

18 Simpson A, Hodges R, Higgins M. Improving communication in obstetrics practice. JObstet Gynaecol Can 2016;38(10): 961-964

19 Curry LA, Nembhard IM, Bradley EH. Qualitative and mixed methods provide unique contributions to outcomes research. Circulation 2009;119(10):1442-1452

20 Farag S, Chyjek K, Chen KT. Identification of iPhone and iPad applications for obstetrics and gynecology providers. Obstet Gynecol 2014;124(05):941-945

21 Rippen HE, Pan EC, Russell C, Byrne CM, Swift EK. Organizational framework for health information technology. Int J Med Inform 2013;82(04):e1-e13 
22 Liu X, Sutton PR, McKenna R, et al. Evaluation of secure messaging applications for a health care system: a case study. Appl Clin Inform 2019;10(01):140-150

23 Tsega S, Kalra A, Sevilla CT, Cho HJ. A bottom-up approach to encouraging sustained user adoption of a secure text messaging application. Appl Clin Inform 2019;10(02):326-330

24 Hagedorn PA, Kirkendall ES, Spooner SA, Mohan V. Inpatient communication networks: leveraging secure text-messaging platforms to gain insight into inpatient communication systems. Appl Clin Inform 2019;10(03):471-478

25 Haun JN, Hathaway W, Chavez M, et al. Clinical practice informs secure messaging benefits and best practices. Appl Clin Inform 2017;8(04):1003-1011

26 Moriates C, Wong BM. High-value care programmes from the bottom-up... and the top-down. BMJ Qual Saf 2016;25(11): 821-823 